\title{
X-Rays from Radio Millisecond Pulsars: Comptonized Thermal Radiation
}

\section{Citation}

Bogdanov, Slavko, Jonathan E. Grindlay, and George B. Rybicki. 2006. "X-Rays from Radio Millisecond Pulsars: Comptonized Thermal Radiation." The Astrophysical Journal 648 (1): L5558. https://doi.org/10.1086/507827.

\section{Permanent link}

http://nrs.harvard.edu/urn-3:HUL.InstRepos:41399947

\section{Terms of Use}

This article was downloaded from Harvard University's DASH repository, and is made available under the terms and conditions applicable to Other Posted Material, as set forth at http:// nrs.harvard.edu/urn-3:HUL.InstRepos:dash.current.terms-of-use\#LAA

\section{Share Your Story}

The Harvard community has made this article openly available.

Please share how this access benefits you. Submit a story.

Accessibility 


\title{
X-RAYS FROM RADIO MILLISECOND PULSARS: COMPTONIZED THERMAL RADIATION
}

\author{
Slavko Bogdanov, Jonathan E. Grindlay, and George B. Rybicki \\ Harvard-Smithsonian Center for Astrophysics, 60 Garden Street, Cambridge, MA 02138; sbogdanov@cfa.harvard.edu, \\ josh@cfa.harvard.edu,grybicki@cfa.harvard.edu \\ Received 2006 May 10; accepted 2006 July 20; published 2006 August 21
}

\begin{abstract}
$\mathrm{X}$-ray emission from many rotation-powered millisecond pulsars (MSPs) is observed to be of predominantly thermal nature. In PSR J0437-4715, the nearest MSP known, an additional faint power-law tail is observed above $2.5 \mathrm{keV}$, commonly attributed to nonthermal magnetospheric radiation. We propose that the hard emission in this and other similar MSPs is instead due to weak Comptonization of the thermal (blackbody or hydrogen atmosphere) polar cap emission by energetic electrons/positrons of small optical depth presumably in the pulsar magnetosphere and wind. This spectral model implies that all soft X-rays are of purely thermal origin, which has profound implications in the study of neutron star structure and fundamental pulsar physics.
\end{abstract}

Subject headings: pulsars: general — pulsars: individual (PSR J0437-4715) — stars: neutron —X-rays: stars

\section{INTRODUCTION}

As the nearest and brightest radio millisecond pulsar (MSP), PSR J0437-4715 (Johnston et al. 1993; van Straten et al. 2001; Hotan et al. 2006) has been the best studied in the X-ray domain. ROSAT, Chandra, and XMM-Newton observations (Zavlin \& Pavlov 1998; Zavlin et al. 2002; Zavlin 2006) have revealed that the $0.1-10 \mathrm{keV}$ emission consists of two thermal components, and a faint power-law (PL) tail, with photon index $\Gamma \sim 2$. The latter can only be discerned in the spectrum beyond $2.5 \mathrm{keV}$. Due to the limited spectral information above $\sim 3 \mathrm{keV}$, the nature of this X-ray component is unclear.

In this Letter we examine the plausibility of various emission mechanisms capable of producing the hard X-rays observed from J0437-4715 (hereafter J0437). The Letter is organized as follows. In $\S 2$ we describe the observations used and their analysis, while in $\S 3$ we outline various interpretations of the emission from J0437. In $\S 4$ we present the results of our analysis and offer a discussion and conclusions in $\S 5$.

\section{OBSERVATIONS AND DATA REDUCTION}

We have retrieved archival Chandra and XMM-Newton observations of J0437 to investigate the X-ray emission from this MSP. The Chandra ACIS-S observations were performed on 2000 May 29-30 (25.7 ks effective exposure, 3'.9 off-axis; see Zavlin et al. 2002 for details). We first reprocessed the level 1 event files using the most recent calibration release and the CIAO 3.3 software package. ${ }^{1}$ The data were then filtered to the nominal Chandra energy band $(0.3-8 \mathrm{keV})$. The source counts were obtained from a 5 " circular region centered on the radio position of the MSP. This circle encloses $>90 \%$ of the total energy.

The XMM-Newton data consist of a 70 ks observation with the EPIC-MOS1/2 detectors in full-frame mode and EPIC-pn instrument in fast-timing mode obtained on 2002 October 9 (see Zavlin 2006). The raw data were reprocessed using the emchain and epchain pipelines of the SAS version 6.5.0 software $^{2}$ and were screened for instances of high background flares, which were subsequently removed. In the case of MOS1/ 2 , the source counts were extracted from $70^{\prime \prime}$ circles, which en-

\footnotetext{
${ }^{1}$ Chandra Interactive Analysis of Observations, available at http://asc.harvard .edu/ciao.

${ }^{2}$ Science Analysis Software, available at http://xmm.vilspa.esa.es.
}

close $>90 \%$ of the total energy. To minimize background contamination the MOS1/2 data were restricted to the $0.3-8 \mathrm{keV}$ band. For the pn detector, the MSP counts were extracted from CCD columns (28)-(39) and were filtered to $0.5-10 \mathrm{keV}$, where the detector response is best known.

For all observations, the background was obtained from a source-free region near the position of the MSP. The spectral fits were performed in XSPEC version 11.3.1. ${ }^{3}$

\section{ORIGIN OF THE HARD X-RAY TAIL}

The inherent faintness of the PL component from J0437 and the limited bandwidth over which it can be observed pose an obstacle in deciphering the origin of this emission. Nonetheless, using the available data we can gain more insight by examining the plausibility of the various mechanisms for production of the observed hard X-ray tail.

\subsection{Nonthermal Emission}

The most widely accepted model for production of a PL spectrum in pulsars involves nonthermal radiation by relativistic electrons and positrons $\left(e^{ \pm}\right)$in the pulsar magnetosphere. The analyses by Zavlin et al. (2002) and Zavlin (2006) of Chandra and XMM-Newton observations revealed that the hard X-ray tail seen in J0437 is well described by a PL with spectral index $\Gamma=2.0 \pm 0.4$. Under the assumption that this emission is of magnetospheric origin, the PL is expected to extend from the optical/UV and beyond the hard X-ray range ( $>10 \mathrm{keV})$. However, Hubble Space Telescope observations have revealed that extrapolation of the PL to lower energies is inconsistent with the far-ultraviolet (FUV) flux from the MSP, with the lower bound $\Gamma \approx 1.6$ only marginally consistent with the FUV flux (Kargaltsev et al. 2004). The measured FUV flux is otherwise well described by $\sim 10^{5} \mathrm{~K}$ thermal emission from the whole neutron star (NS) surface. Unless a break in the PL exists below $0.1 \mathrm{keV}$, the apparent discrepancy casts doubt on the validity of the nonthermal model.

Becker \& Trümper (1999) have proposed that all X-rays from J0437 and other nearby MSPs could be due to nonthermal emission processes in the magnetosphere. However, on the basis of more recent observations of these MSPs, we conclude that this

\footnotetext{
${ }_{3}^{3}$ See http://heasarc.gsfc.nasa.gov/docs/xanadu/xspec.
} 


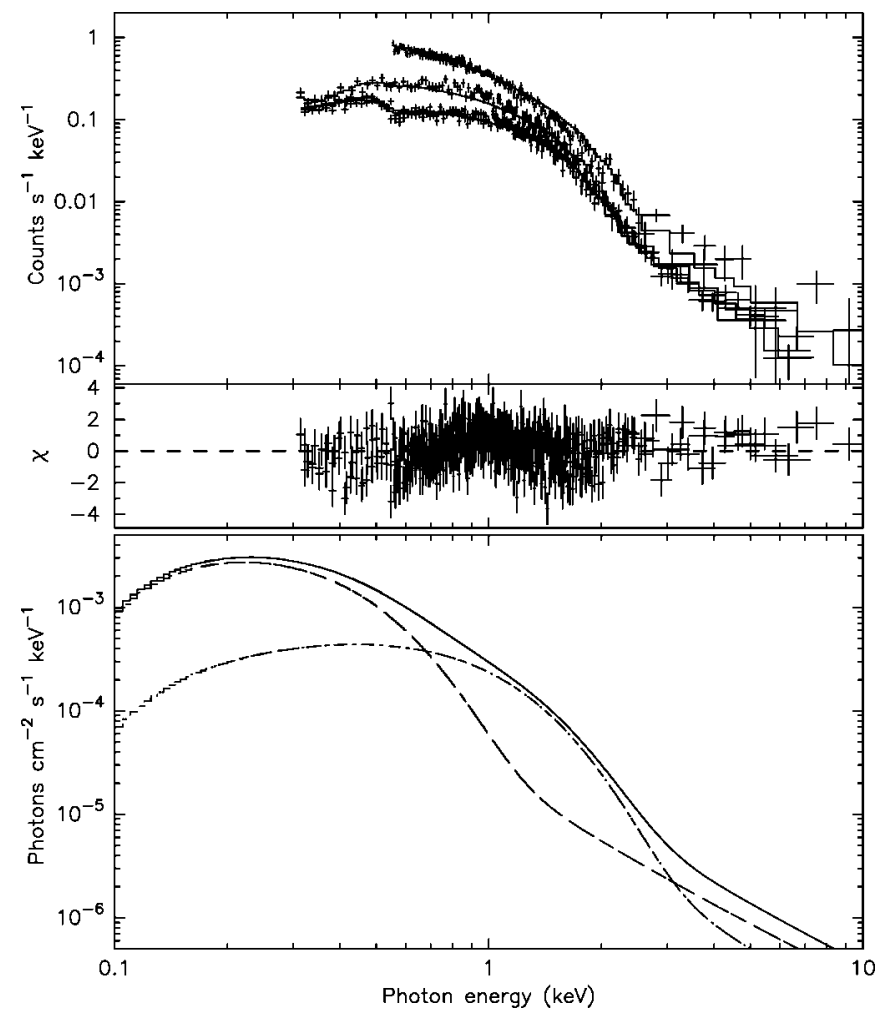

FIG. 1.-Top: Chandra ACIS-S and XMM-Newton EPIC-MOS1/2 and EPICpn spectra of J0437 fitted with a two-temperature Comptonized blackbody model (compbb). The lower panel shows the best-fit residuals. Bottom: Model spectra used in the fit. The solid line is the total spectrum, while the dashed and dot-dashed lines show the individual emission components (see text for best-fit parameters).

interpretation is untenable. If the emission were indeed of purely nonthermal character, then such a broken PL spectrum grossly overestimates the observed optical/UV fluxes (Koptsevich et al. 2003; Mignani \& Becker 2004; Kargaltsev et al. 2004). Furthermore, no breaks are observed in the X-ray spectra of the luminous MSPs J0218+4232, B1821-24, and B1937+21, which show purely nonthermal pulsed radiation (see, e.g., Kuiper et al. 2002; Becker et al. 2003; Mineo et al. 2004).

An alternative source of nonthermal X-rays is nebular synchrotron emission from a shock formed due to interaction between the wind from the MSP and the companion star. Such a shock is likely present in other binary MSPs, namely, PSRs B1957+20 (Stappers et al. 2003), J0024-7204W (Bogdanov et al. 2005), and J1740-5340 (J. E. Grindlay et al. 2006, in preparation). However, for J0437, the strength of the pulsar wind $\dot{E} \sim 4 \times 10^{33} \mathrm{ergs} \mathrm{s}^{-1}$ and the orbital separation ${ }^{4}$ of $a=1.2 \times 10^{12} \mathrm{~cm}$ do not favor this interpretation as the expected X-ray luminosity from the resulting shock is substantially lower than that observed. Specifically, assuming a radius $\sim 1.5 \times 10^{9} \mathrm{~cm}$ for the $0.24 M_{\odot}$ white dwarf (WD) companion (van Straten et al. 2001), we find that the total incident power on the WD from the pulsar wind is $\sim 10^{27} \mathrm{ergs} \mathrm{s}^{-1}$, more than 2 orders of magnitude lower than the observed luminosity of the hard emission $\left(L_{\mathrm{X}} \approx 7 \times 10^{29} \mathrm{ergs} \mathrm{s}^{-1}\right.$ for $\left.0.1-7 \mathrm{keV}\right)$. The large discrepancy can be reduced in a more contrived scenario,

\footnotetext{
${ }^{4}$ In Zavlin et al. (2002) the semimajor axis of the MSP $\left(a_{1}\right)$ is erroneously quoted as being the distance between the MSP and its companion $(a=$ $\left.a_{1}+a_{2}\right)$. Nevertheless, their conclusion that an intrabinary shock can be ruled out is correct.
}

involving a highly anisotropic wind, confined to the orbital plane in a beam covering $<0.15 \%$ of the sky. Alternatively, the companion may be bloated or surrounded by a tenuous envelope of gas that interacts with the MSP wind. However, this assertion is in conflict with the observed optical properties of J0437, which are entirely consistent with that of a $\sim 0.2 M_{\odot}$ He WD (see, e.g., Bailyn 1993). Therefore, we conclude that an intrabinary shock is not a significant source of X-ray emission in the J0437 system.

\subsection{Thermal Emission}

In a purely thermal scenario the hard emission from J0437 may originate from a very small hot spot on the polar cap with a radius of order a few meters, radiating at temperatures in excess of $8 \times 10^{6} \mathrm{~K}$. However, no current MSP polar cap heating model predicts such peculiarly high temperatures and small emission areas, making this model rather implausible.

\subsection{Comptonized Thermal Emission}

The hard X-ray tail can be naturally produced by reprocessing of the thermal emission via inverse Compton scattering (ICS) by an optically thin $(\tau<1)$ hot plasma. As a thermal photon emitted at the NS surface propagates through the tenuous magnetospheric plasma and the pulsar wind, it may undergo ICS by energetic $e^{ \pm}$. In this regime, repeated scatterings of the thermal seed photons result in a PL distribution of photons (see Rybicki \& Lightman 1979; Nishimura et al. 1986). If we assume a Goldreich-Julian (GJ) charged particle density in the MSP magnetosphere, we find $n \sim 0.07 B / P=4 \times 10^{9} \mathrm{~cm}^{-3}$ (Goldreich \& Julian 1969), with $B \sim 10^{8} \mathrm{G}$ and $P=5.76 \mathrm{~ms}$. From here, the scattering optical depth is simply $\tau_{s}=n L \sigma$, where $L$ is the path length through the scattering medium and $\sigma$ is the scattering cross section. If we assume $L$ to be of order the light cylinder radius $\left(r_{\mathrm{lc}}=c P / 2 \pi=275 \mathrm{~km}\right)$ and $\sigma$ to be the Thomson cross section, we obtain a value of $\tau_{s} \sim 7 \times 10^{-8}$, too low to have a measurable effect on the spectrum. Thus, if the PL tail in J0437-4715 is indeed due to Comptonization, an enhanced density $\left(n \gg n_{\mathrm{GJ}}\right)$ and/or $L \gg r_{\mathrm{lc}}$ are required to produce a sufficient scattering optical depth. The plasma density may, in fact, be substantially larger than the GJ density, for example, due to pair production cascades by high-energy $\gamma$-rays. In addition, scattering of the thermal photons may take place in the pulsar wind as well, up to distances $L \gg r_{\text {Ic }}$ from the MSP. Due to the lack of any observational information on the true $n$ near the pulsar as well as the maximum extent of the scattering region $(L)$, at present we are only able to place a conservative lower limit on the optical depth of the scattering plasma, $\tau_{s} \geq 7 \times 10^{-8}$, obtained in the calculation above.

A major advantage of this model is that, unlike the case of a nonthermal (magnetospheric) emission PL, it does not extend below $\sim 1 \mathrm{keV}$ (see Fig. 1), thus alleviating any discrepancies with optical/UV data. Therefore, on the basis of the available observational evidence and the relative simplicity of the Comptonization model, we deem this interpretation the most plausible.

\section{RESULTS}

We have examined the effect of ICS on the thermal emission from MSPs for both a blackbody (BB) and neutron star hydrogen atmosphere (NSA; see Romani 1987; Zavlin et al. 1996) model. For the BB case we employed the compbb model in XSPEC (see Nishimura et al. 1986, for details). This particular model 


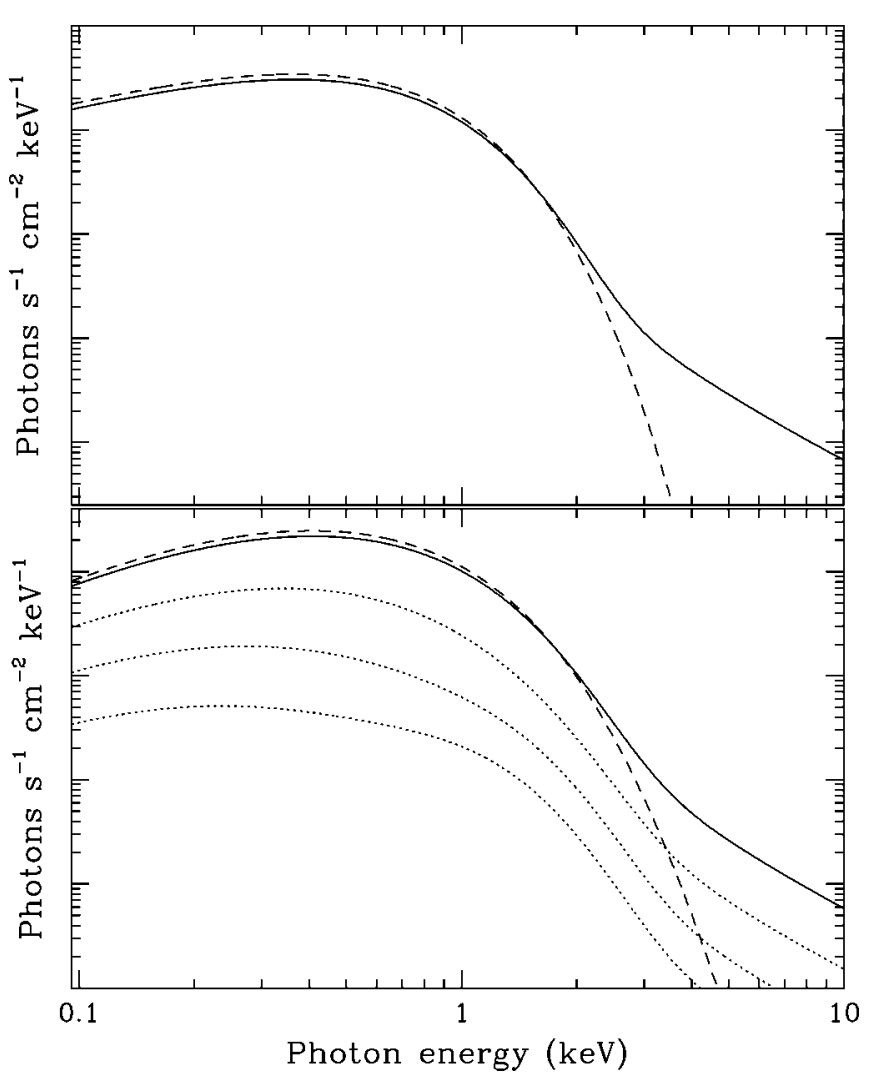

FIG. 2.-Top: Example Comptonized BB model spectrum for $k T_{\text {eff }}=0.23 \mathrm{keV}$, $k T_{e}=100 \mathrm{keV}$, and $\tau=0.1$. Bottom: Comptonized NSA model spectrum for $T_{\text {eff }}=1.3 \times 10^{6} \mathrm{~K}, k T_{e}=100 \mathrm{keV}$, and $\tau=0.1$. The photon energies have been corrected for the gravitational redshift assuming $R=10 \mathrm{~km}$ and $M=1.4 M_{\odot}$. The dotted lines correspond to different viewing angles relative to the surface normal ( $\cos \theta=0.5,0.25$, and 0.1 , from top to bottom), while the solid line is for $\cos \theta=1$. In both plots the dashed line shows the initial thermal photon distribution. The flux normalization is arbitrary.

assumes a plane-parallel, semi-infinite scattering medium with the source of thermal photons at the bottom, which greatly simplifies the treatment of Comptonization in the low optical depth regime. It is valid over the range $E \ll k T_{e}<m c^{2}$, where $E$ is the initial photon energy, $T_{e}$ is the scattering $e^{ \pm}$temperature, and $m c^{2}$ is the electron rest energy. Figure 1 shows the X-ray spectrum of J0437 fitted with a two-temperature Comptonized BB model using compbo. Throughout the spectral fits we fixed the hydrogen column density toward the MSP to $N_{\mathrm{H}}=2 \times 10^{19} \mathrm{~cm}^{-2}$ and electron temperature to $k T_{e}=150 \mathrm{keV}$. The best-fit parameters we obtain are $T_{1}=(2.93 \pm 0.03) \times 10^{6} \mathrm{~K}, R_{1}=44 \pm 11 \mathrm{~m}, \tau_{1}=$ $0.06 \pm 0.02, T_{2}=(1.16 \pm 0.01) \times 10^{6} \mathrm{~K}, R_{2}=300 \pm 40 \mathrm{~m}$, and $\tau_{2}=0.09 \pm 0.01$. The corresponding luminosity in the $0.3-$ $10 \mathrm{keV}$ is found to be $L_{\mathrm{X}} \approx 3 \times 10^{30} \mathrm{ergs} \mathrm{s}^{-1}$. All uncertainties quoted above are $1 \sigma$. The minimum value of $\chi_{v}^{2}=1.36$ (for 464 degrees of freedom) was found to be identical to that in the case of the $\mathrm{BB}+\mathrm{BB}+\mathrm{PL}$ model. Given the uncertainties in the crosscalibration of the different detectors, this value of $\chi_{\nu}^{2}$ indicates an acceptable fit. As is generally the case for ICS, $T_{e}$ and $\tau$ are strongly correlated, so a good fit was obtained for a wide range of $k T_{e}$ and $\tau$. Note that although the derived $\tau_{1}$ and $\tau_{2}$ are consistent with being identical, they may, in fact, differ depending on the precise geometry of the Comptonizing layer(s).

The compbo model assumes a thermal distribution of scattering $e^{ \pm}$. However, in the vicinity of the pulsar it is difficult to maintain a thermal population of $e^{ \pm}$due to the strong induced

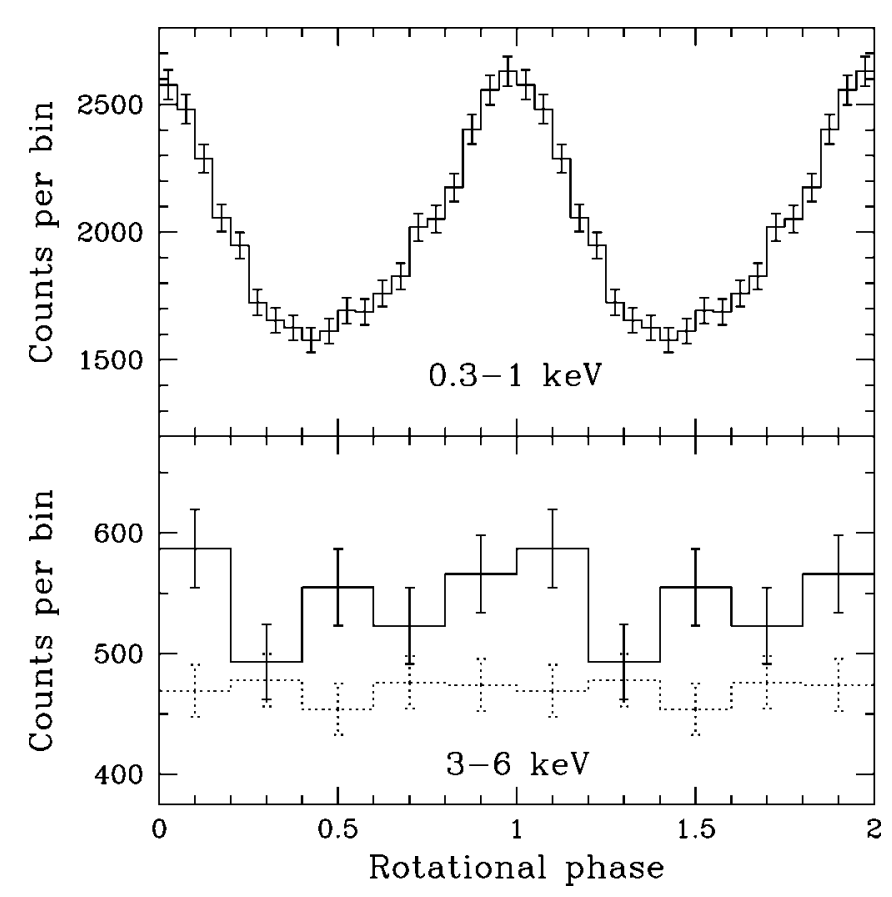

FIG. 3.-XMM-Newton EPIC-pn light curves of J0437 in the $0.3-1 \mathrm{keV}$ (top) and 3-6 keV (bottom) bands. The dotted line in the bottom panel shows the background level. Two cycles are shown for clarity.

electric field. In reality, these particles most likely follow a nonthermal (PL) distribution over a certain range of energies. Notwithstanding, the exact energy distribution of the $e^{ \pm}$has no bearing on the validity of this model and our results, since in both scenarios, multiple scatterings result in a PL distribution of photons.

To study the effect of Comptonization on the emission from a NSA we used the unmagnetized hydrogen atmosphere model from Heinke et al. (2006; see also McClintock et al. 2004) and the Comptonization algorithm of Nishimura et al. (1986). As expected, we have found that the NSA model yields a qualitatively similar spectrum to that of the BB case (see Fig. 2). However, crucial differences do exist, stemming from the inherently anisotropic and energy-dependent emission pattern of a NSA (Zavlin et al. 1996). In particular, at a given viewing angle, the intensity of the NSA radiation is subject to limb darkening as well as a projection effect. The scattered photons, on the other hand, probably have a different (more isotropic) angular distribution. This suggests that, depending on the viewing geometry, the observed flux may show variations in the relative contribution from the Comptonized components.

It is important to stress that the exact geometry and location of the scattering medium relative to the MSP polar caps is unknown and cannot be determined from the time-integrated spectra alone. In principle, these properties can be inferred from the modulation of the Comptonized radiation as a function of the spin phase of the MSP. To investigate the temporal behavior of the PL tail, we used the timing data from the XMM-Newton EPIC-pn detector. Figure 3 shows light curves of J0437 folded at the MSP spin period for the $0.3-1 \mathrm{keV}$ and 3-6 keV energy ranges. In the Comptonization scenario, these two bands contain purely (unscattered) thermal and just Comptonized photons, respectively. Unfortunately, due to the limited count statistics and the dominant instrument and sky backgrounds for $>3 \mathrm{keV}$, at present we cannot provide any meaningful con- 
straints on the properties of the Comptonizing medium. We are, however, able to place a limit on the pulsed fraction (rms; see Dhillon et al. 2005) of $\$ 50 \%$ for $>3 \mathrm{keV}$. This value further constrains a nonthermal magnetospheric origin since such emission is expected to be strongly pulsed.

\section{DISCUSSION AND CONCLUSION}

We have demonstrated that the X-ray emission from the nearby MSP J0437 in the $0.1-10 \mathrm{keV}$ band is well described by a two-temperature weakly Comptonized thermal spectrum. This model provides a viable and relatively simple alternative to the nonthermal magnetospheric emission model. Since ICS is the primary mechanism for pair production and polar cap heating in the Harding \& Muslimov (2002) model of highenergy emission from MSPs, the presence of a hard Comptonized tail would provide a strong confirmation that this mechanism does indeed operate in MSP magnetospheres.

Here we have considered a fairly simplistic Comptonization model that does not fully take into account the spatial and energy distribution of the scattering particles. A more elaborate and realistic model that incorporates details regarding the geometry and physical properties of the scattering medium is beyond the scope of this Letter but will be addressed in the future.

Note that the shapes of the spectra in the nonthermal magnetospheric and Comptonization cases are indistinguishable in the $0.1-10 \mathrm{keV}$ band. The primary difference arises in the optical/UV range, where only the former is expected. As discussed above, for J0437 the nonthermal model has been found to be only marginally consistent with FUV observations. The detection of a hard X-ray tail from other nearby MSPs, for example, PSRs J0030+0451 and J2124-3358, would provide a more definitive test of the two interpretations (the existence of a hard X-ray component cannot be ascertained in present X-ray observations of these MSPs due to the limited counts). For these solitary MSPs, the absence of a stellar companion has allowed ultradeep optical observations, which have found no emission down to $V \sim 28$ (Koptsevich et al. 2003; Mignani \& Becker 2004).

The Comptonization model proposed in this Letter implies that all soft emission $(<1 \mathrm{keV})$ from $\mathrm{J} 0437-4715$ is purely thermal (unscattered) radiation. This has profound ramifications in the study of fundamental NS properties since thermal emission from the surface of neutron stars reveals important information regarding the basic properties of this class of compact objects. Pavlov \& Zavlin (1997) have shown that X-ray spectral and timing observations of MSPs can be used to measure fundamental NS parameters such as the mass-to-radius ratio of the underlying NS. In addition, the polar caps represent "footprints" of the pulsar magnetic field, allowing constraints on the global field geometry. Finally, since the properties of the hard emission component are determined by the energy distribution and optical depth of the scattering $e^{ \pm}$, study of this radiation may also serve as a valuable probe of the particles populating the pulsar magnetosphere and wind. An attempt to constrain the properties of J0437 outlined above is currently under way and will be presented in a future paper.

This work was supported in part by NASA grant AR67010X. The research presented here has made use of the NASA Astrophysics Data System (ADS).

\section{REFERENCES}

Bailyn, C. D. 1993, ApJ, 411, L83

1999, A\&A, 341, 803

Becker, W., et al. 2003, ApJ, 594, 798

Bogdanov, S., Grindlay, J. E., \& van den Berg, M. 2005, ApJ, 630, 1029

Dhillon, V. S., Marsh, T. R., Hulleman, F., van Kerkwijk, M. H., Shearer, A.,

Littlefair, S. P., Gavriil, F. P., \& Kaspi, V. M. 2005, MNRAS, 363, 609

Goldreich, P., \& Julian, W. H. 1969, ApJ, 157, 869

Harding, A. K., \& Muslimov, A. G. 2002, ApJ, 568, 862

Heinke, C. O., Rybicki, G. B., Narayan, R., \& Grindlay, J. E. 2006, ApJ, 644, 1090

Hotan, A. W., Bailes, M., \& Ord, S. M. 2006, MNRAS, 369, 1502

Johnston, S., et al. 1993, Nature, 361, 613

Kargaltsev, O. Y., Pavlov, G. G., \& Romani, R. W. 2004, ApJ, 602, 327

Koptsevich, A. B., Lundqvist, P., Serafimovich, N. I., Shibanov, Yu. A., \& Sollerman, J. 2003, A\&A, 400, 265

Kuiper, L., Hermsen, W., Verbunt, F., Ord, S., Stairs, I., \& Lyne, A. 2002, ApJ, 577, 917

McClintock, J. E., Narayan, R., \& Rybicki, G. B. 2004, ApJ, 615, 402
Mignani, R. P., \& Becker, W. 2004, Adv. Space Res., 33, 616

Mineo, T., Cusumano, G., Massaro, E., Becker, W., \& Nicastro, L. 2004, A\&A, 423, 1045

Nishimura, J., Mitsuda, K., \& Itoh, M. 1986, PASJ, 38, 819

Pavlov, G. G., \& Zavlin, V. E. 1997, ApJ, 490, L91

Romani, R. W. 1987, ApJ, 313, 718

Rybicki, G. B., \& Lightman, A. P. 1979, Radiative Processes in Astrophysics (New York: Wiley)

Stappers, B. W., Gaensler, B. M., Kaspi, V. M., van der Klis, M., \& Lewin, W. H. G. 2003, Science, 299, 1372

van Straten, W., Bailes, M., Britton, M., Kulkarni, S. R, Anderson, S. B., Manchester, R. N., \& Sarkissian, J. 2001, Nature, 412, 158

Zavlin, V. E. 2006, ApJ, 638, 951

Zavlin, V. E., \& Pavlov, G. G. 1998, A\&A, 329, 583

Zavlin, V. E., Pavlov, G. G., Sanwal, D., Manchester, R. N., Trümper, J., Halpern, J. P., \& Becker, W. 2002, ApJ, 569, 894

Zavlin, V. E., Pavlov, G. G., \& Shibanov, Yu. A. 1996, A\&A, 315, 141 